LAWRENCE LIVERMORE NAT IO N A L LABORATORY

Nd-Doped Laser Glass

Spectroscopic and Physical Properties

S. E. Stokowski, R. A. Saroyan, M. J. Weber

November 22, 2004 
This document was prepared as an account of work sponsored by an agency of the United States Government. Neither the United States Government nor the University of California nor any of their employees, makes any warranty, express or implied, or assumes any legal liability or responsibility for the accuracy, completeness, or usefulness of any information, apparatus, product, or process disclosed, or represents that its use would not infringe privately owned rights. Reference herein to any specific commercial product, process, or service by trade name, trademark, manufacturer, or otherwise, does not necessarily constitute or imply its endorsement, recommendation, or favoring by the United States Government or the University of California. The views and opinions of authors expressed herein do not necessarily state or reflect those of the United States Government or the University of California, and shall not be used for advertising or product endorsement purposes.

This work was performed under the auspices of the U.S. Department of Energy by University of California, Lawrence Livermore National Laboratory under Contract W-7405-Eng-48. 
M-95, Rev. 2 Volume 1

\section{Nd-Doped Laser Glass Spectroscopic and Physical Properties}

S. E. Stokowski
R. A. Saroyan
M. J. Weber

Manuscript date: November 1981 
Current status: Research sample

Melt:

K-824, December 1976

\section{COMPOSITION}

\begin{tabular}{lcc}
\hline Compound & Mole \% & Weight \% \\
\hline $\mathrm{Ta}_{2} \mathrm{O}_{5}$ & 17.0 & 58.79 \\
$\mathrm{MgO}$ & 31.5 & 9.6 \\
$\mathrm{SiO}_{2}$ & 38.0 & 17.26 \\
$\mathrm{BaO}$ & 13.0 & 15.07 \\
$\mathrm{Nd}_{2} \mathrm{O}_{3}$ & 0.5 & 1.27
\end{tabular}

$\begin{array}{lcc}\text { Measured neodymium concentration } & - & \text { wt \% } \mathrm{Nd}_{2} \mathrm{O}_{3} \\ \text { Neodymium number density } \rho_{\mathrm{N}} & 2.378 \times 10^{20} & \mathrm{~cm}^{-3}\end{array}$

Compositional series:

\section{PROPERTY SUMMARY}

Peak stimulated emission cross section Calculated radiative lifetime Zero-concentration lifetime $\mathrm{Nd}^{3+}$ concentration $@ \tau_{0} / 2$ Nonlinear refractive index coefficient Nonlinear refractive index Refractive index at peak-fluorescence wavelength Peak-fluorescence wavelength Linewidth (FWHM) Linewidth (effective) Density

$\begin{array}{lll}\sigma_{\mathrm{P}} & 2.4 \times 10^{-20} & \mathrm{~cm}^{2} \\ \tau_{\mathrm{R}}^{-} & -274 & \mu \mathrm{sec} \\ \tau_{0} & - & \mu \mathrm{sec} \\ \mathrm{Q}^{2} & - & \mathrm{cm}^{-3} \\ \gamma & 8.48 \times 10^{-20} & \mathrm{~m}^{2} \cdot \mathrm{W}^{-1} \\ \mathrm{n}_{2} & 3.44 \times 10^{-13} & \mathrm{esu} \\ \mathrm{n}\left(\lambda_{\mathrm{p}}\right) & 1.70327 & \\ \lambda_{\mathrm{p}} & 1064.5 & \mathrm{~nm} \\ \Delta \lambda & 38.20 & \mathrm{~nm} \\ \Delta \lambda_{\text {eff }} & 42.64 & \mathrm{~nm} \\ \rho & 5.23 & \mathrm{~g} \cdot \mathrm{cm}^{-3}\end{array}$




\section{ABSORPTION PROPERTIES}
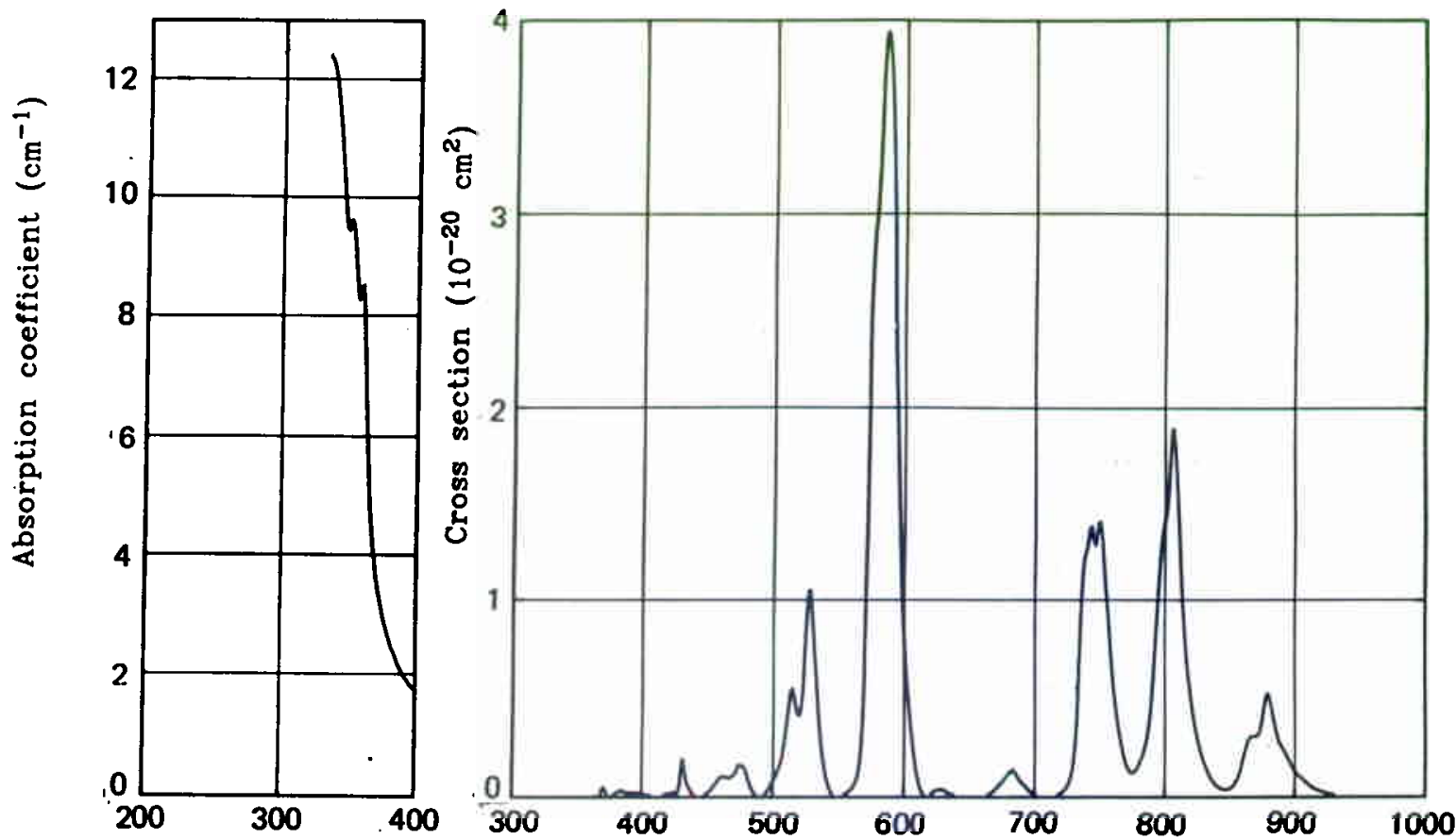

Wavelength $(\mathrm{nm})$

Integrated absorption cross section relative

to ED-2 from 400 to $950 \mathrm{~nm}$

1.32

Absorption efficiency coefficients for $15-\mathrm{mm}$

xenon flashlamp at $1000 \mathrm{~A} \cdot \mathrm{cm}^{-2}$

(400 to $940 \mathrm{~nm}$ ):

$\varepsilon=A\left[1-\mathrm{B} \exp \left(-\mathrm{C} \rho_{\mathrm{N}} l\right)-(1-\mathrm{B}) \exp \left(-\mathrm{D} \rho_{\mathrm{N}} l\right)\right]$

where $\rho_{\mathrm{N}} l$ is in $10^{20} \mathrm{Nd}$ ions $\cdot \mathrm{cm}^{-2}$

$\dot{A}=\overrightarrow{0} .109258$

$\mathrm{B}=\mathbf{0 . 6 9 1 3 3 0}$

$C=0.154631$

$D=1.328205 \mathrm{~cm}^{2}$

Judd-Ofelt parameters:

$\Omega_{2}$
$\Omega_{4}$
$\Omega_{6}$

$7.0 \pm 0.3 \times 10^{-20}$

$4.6 \pm 0.4 \times 10^{-20}$

$4.7 \pm 0.2 \times 10^{-20}$

$\mathrm{cm}^{2}$

$\mathrm{cm}^{2}$

$\mathrm{cm}^{2}$ 


\section{FLUORESCENCE PROPERTIES}

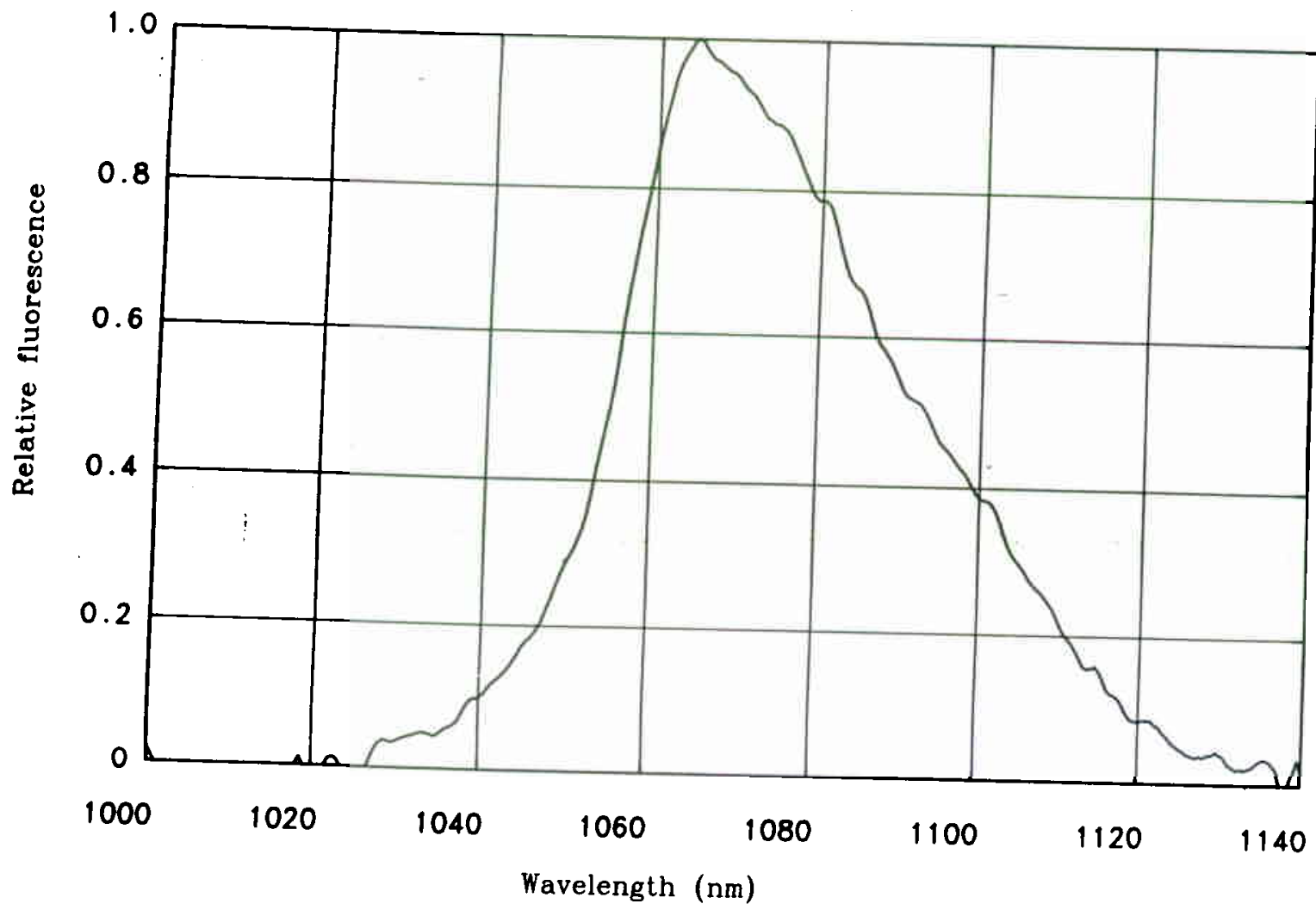

Peak-fluorescence wavelength

Linewidth (FWHM)

Linewidth (effective)

Peak stimulated emission cross section

Calculated radiative lifetime

Zero-concentration lifetime

$\mathrm{Nd}^{3+}$ concentration $@ \tau_{0} / 2$

Branching ratios:

$$
\left({ }^{4} \mathrm{~F}_{3 / 2} \rightarrow{ }^{4} \mathrm{I}_{\mathrm{J}}\right) \mathrm{J}=9 / 2,11 / 2,13 / 2,15 / 2
$$

$\lambda_{\mathrm{p}}$
$\Delta \lambda$
$\Delta \lambda_{\text {eff }}$
$\sigma_{\mathrm{P}}$
$\tau_{\mathrm{R}}$
$\tau_{0}$
$Q$

$0.430,0.477,0.090,0.004$

Measured decay properties: three e-folding times

$190,214,230$

$\mu \mathrm{sec}$

Decay function: $I / I_{0}=A \exp (-B t)+C \exp (-D t)+E \exp (-F t)$
$A=-$
$B=-$
$\sec ^{-1}$
$\mathrm{C}=-$
$\mathrm{D}=-$
$\sec ^{-1}$
$\mathrm{E}=-$
$F=-$
$\sec ^{-1}$ 


\section{OPTICAL PROPERTIES}

Refractive index at sodium $D$ line

Refractive index at peak fluorescence

Abbe number, $\left(n_{D}-1\right) /\left(n_{F}-n_{C}\right)$

Index temperature cófficient

$\begin{array}{ll}\mathrm{n}_{\mathrm{D}} & 1.72 \\ \mathrm{n}\left(\lambda_{\mathrm{p}}\right) & 1.70327 \\ \nu & 45.0\end{array}$

$\partial \mathrm{n} / \partial \mathrm{T} \quad-$

${ }^{\circ} \mathrm{C}^{-1}$

Thermal coefficient of optical path length

$$
\partial \mathrm{s} / \partial \mathrm{T}
$$

$-$

$\gamma$ calc

$\gamma$, meas

Nonlinear refractive index

Stress optic coefficient

$\mathrm{n}_{2}$ calc

$\mathrm{n}_{2}$ meas

$\Delta \mathrm{B}$

$$
\begin{aligned}
& 8.48 \times 10^{-20} \\
& - \\
& 3.44 \times 10^{-13} \\
& - \\
& -
\end{aligned}
$$

${ }^{\circ} \mathrm{C}^{-1}$

$\mathrm{m}^{2} \cdot \mathrm{w}^{-1}$

$\mathrm{m}^{2} \cdot \mathrm{w}^{-1}$

esu

esu

$\mathrm{nm} \cdot \mathrm{cm} \cdot \mathrm{kg}^{-1}$

\section{THERMAL PROPERTIES}

Thermal expansion coefficient

Specific heat at constant pressure

Thermal heat conductivity

Softening point

Transformation point

$\begin{array}{lll}\alpha & - & { }^{\circ} \mathrm{C}^{-1} \\ \mathrm{C}_{\mathrm{P}} & - & \mathrm{J} \cdot \mathrm{cm}^{-3} \cdot{ }^{\circ} \mathrm{C}^{-1} \\ \kappa & - & \mathrm{W} \cdot \mathrm{m}^{-1} \cdot{ }^{\circ} \mathrm{C}^{-1} \\ \mathrm{~T}_{\mathrm{y}} & - & { }^{\circ} \mathrm{C} \\ \mathrm{T}_{\mathrm{g}} & - & { }^{\circ} \mathrm{C}\end{array}$

\section{MECHANICAL PROPERTIES}

Density

Knoop hardness

Young's modulus

p $\quad 5.23$

$\mathrm{g} \cdot \mathrm{cm}^{-3}$

$\mathrm{K}_{\mathrm{H}}$

$-$

$\mathrm{kg} \cdot \mathrm{mm}^{-2}$

Shear modulus

E

G

Poisson ratio

$-$

$-$

$\mathrm{kg} \cdot \mathrm{mm}^{-2}$

$\mathrm{kg} \cdot \mathrm{mm}^{-2}$

\section{SOLUBILITY}

Stability in water

Stability in acid

\section{REFERENCES and NOTES}


Current status: Research sample

Melt:

EK-2236, 1973

\section{COMPOSITION}

\begin{tabular}{lrl}
\hline Compound & Mole \% & Weight \% \\
\hline $\mathrm{Bi}_{2} \mathrm{O}_{3}$ & 24.21 & 60.0 \\
$\mathrm{CdO}$ & 43.93 & 30.0 \\
$\mathrm{SiO}_{2}$ & 31.29 & 10.0 \\
$\mathrm{Nd}_{2} \mathrm{O}_{3}$ & 0.56 & 1.0
\end{tabular}

$\begin{array}{lll}\text { Measured neodymium concentration } & - & \mathrm{wt} \% \mathrm{Nd}_{2} \mathrm{O}_{3} \\ \text { Neodymium number density } \rho_{\mathrm{N}} & 2.403 \times 10^{20} & \mathrm{~cm}^{-3}\end{array}$

Compositional series:

\section{PROPERTY SUMMARY}

$\begin{array}{llll}\text { Peak stimulated emission cross section } & \sigma_{\mathrm{P}} & -3.6 \times 10^{-20} & \mathrm{~cm}^{2} \\ \text { Calculated radiative lifetime } & \tau_{\mathrm{R}} & 234 & \mu \mathrm{sec} \\ \text { Zero-concentration lifetime } & \tau_{0} & - & \mu \mathrm{sec} \\ \mathrm{Nd}^{3+} \text { concentration } \tau_{0} / 2 & \mathrm{Q} & - & \mathrm{\textrm {cm } ^ { - 3 }} \\ \text { Nonlinear refractive index coefficient } & \gamma & 15.6 \times 10^{-20} & \mathrm{~m}^{2} \cdot \mathrm{W}^{-1} \\ \text { Nonlinear refractive index } & \mathrm{n}_{2} & 6.29 \times 10^{-13} & \mathrm{esu} \\ \text { Refractive index at peak-fluorescence wavelength } & \mathrm{n}\left(\lambda_{\mathrm{p}}\right) & 1.69492 & \\ \text { Peak-fluorescence wavelength } & \lambda_{\mathrm{p}} & 1063.5 & \mathrm{~nm} \\ \text { Linewidth (FWHM) } & \Delta \lambda & 28.43 & \mathrm{~nm} \\ \text { Linewidth (effective) } & \Delta \lambda_{\mathrm{efl}} & 34.57 & \mathrm{~nm} \\ \text { Density } & \rho & 6.711 & \mathrm{~g} \cdot \mathrm{cm}^{-3}\end{array}$




\section{ABSORPTION PROPERTIES}
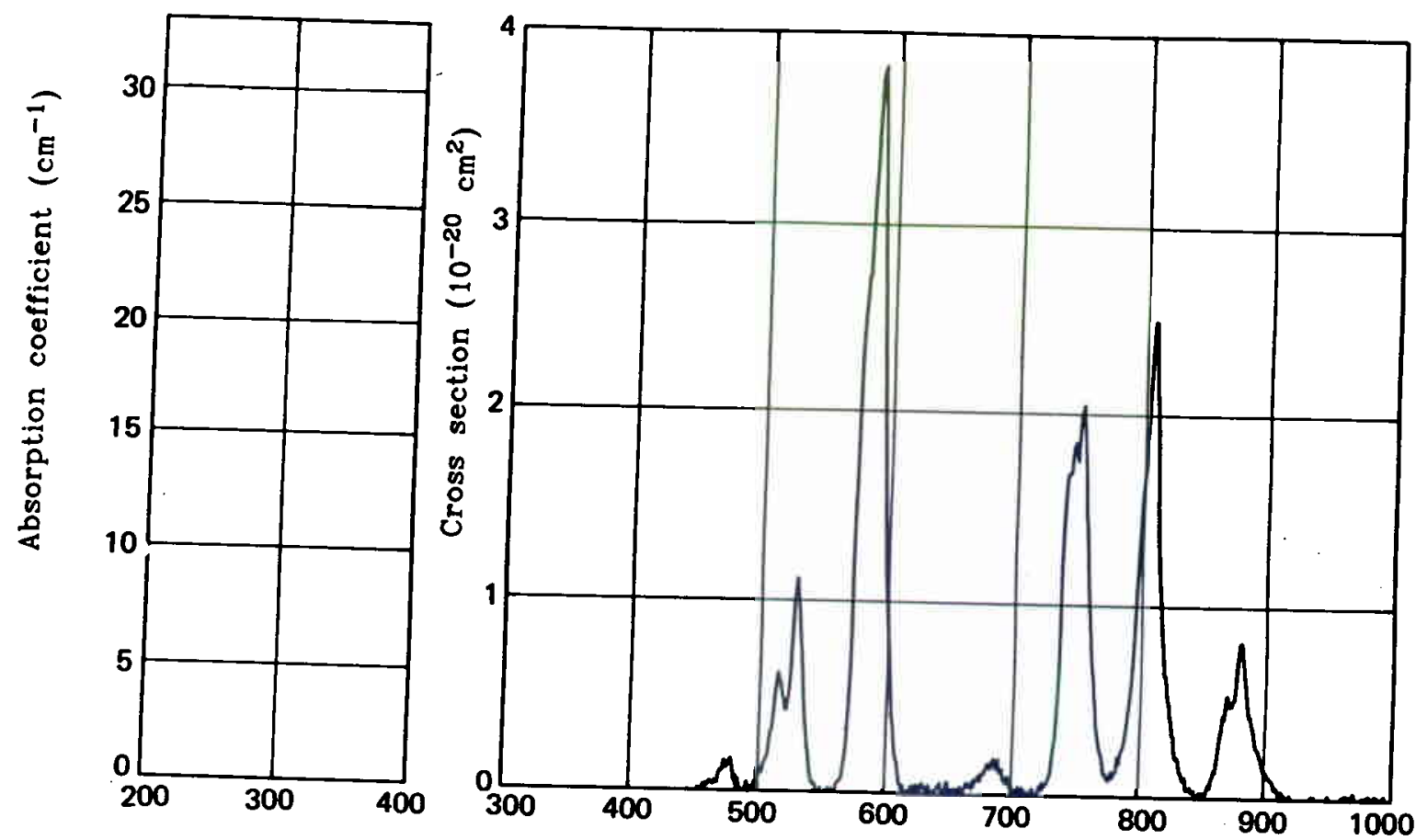

Wavelength $(\mathrm{nm})$

Integrated absorption cross section relative

to $E D-2$ from 400 to $950 \mathrm{~nm}$

Absorption efficiency coefficients for $15-\mathrm{mm}$

xenon flashlamp at $1000 \mathrm{~A} \cdot \mathrm{cm}^{-2}$

(340 to $940 \mathrm{~nm})$ :

$\varepsilon=A\left[1-\mathrm{B} \exp \left(-\mathrm{C} \rho_{N} l\right)-(1-\mathrm{B}) \exp \left(-\mathrm{D} \rho_{N} l\right)\right]$

where $\rho_{N} l$ is in $10^{20} \mathrm{Nd}$ ions $\cdot \mathrm{cm}^{-2}$
$A=0.110932-B=0.669260$
$C=0.159280 \sim D=1.365418 \mathrm{~cm}^{2}$

Judd-Ofelt parameters:

$\begin{array}{ll}\Omega_{2} & 5.4 \pm 0.3 \times 10^{-20} \\ \Omega_{4} & 5.1 \pm 0.5 \times 10^{-20} \\ \Omega_{6} & 5.9 \pm 0.2 \times 10^{-20}\end{array}$

$\mathrm{cm}^{2}$

$\mathrm{cm}^{2}$

$\mathrm{cm}^{2}$ 


\section{FLUORESCENCE PROPERTIES}

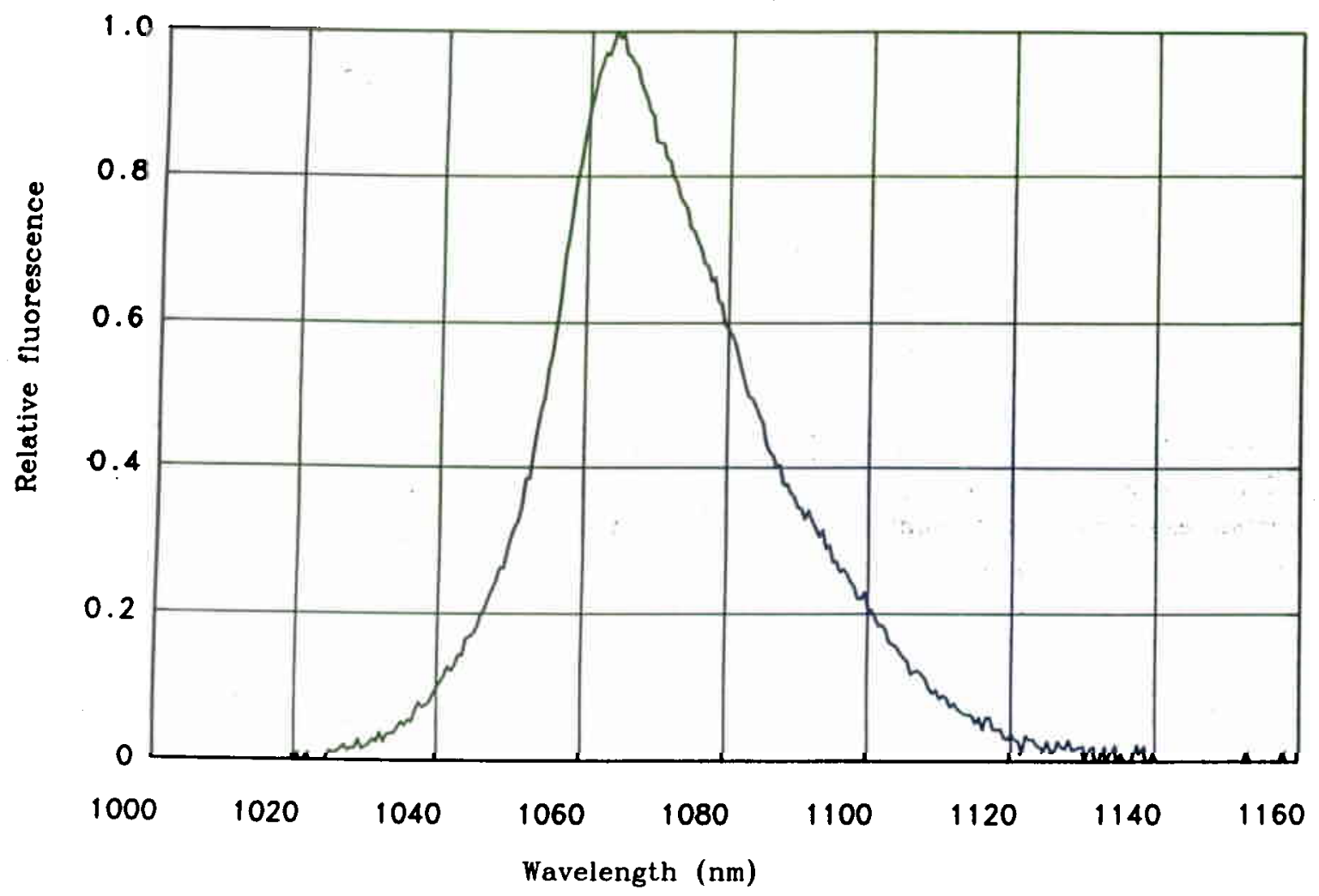

Peak-fluorescence wavelength Linewidth (FWHM)

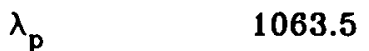

$\mathrm{nm}$ Linewidth (effective)

Peak stimulated emission cross section Calculated radiative lifetime Zero-concentration lifetime $\mathrm{Nd}^{3+}$ concentration $2 \tau_{0} / 2$

Branching ratios:

$$
\left({ }^{4} F_{3 / 2} \rightarrow{ }^{4} I_{J}\right) J=9 / 2,11 / 2,13 / 2,15 / 2 \quad 0.411,0.490,0.095,0.005
$$

Measured decay properties: three e-folding times

Decay function: $I / I_{0}=A \exp (-B t)+C \exp (-D t)+E \exp (-F t)$
$\mathrm{A}=-$
$\mathrm{B}=$
$\sec ^{-1}$
$C=-$
$\mathrm{D}=-$
$\sec ^{-1}$
$\mathrm{E}=$ -
$F=-$
$\sec ^{-1}$ 


\section{OPTICAL PROPERTIES}

Refractive index at sodium $D$ line Refractive index at peak fluorescence Abbe number, $\left(n_{D}-1\right) /\left(n_{F}-n_{C}\right)$ Index temperature coefficient

Thermal coefficient of optical path length

Nonlinear refractive index coefficient

Nonlinear refractive index

Stress optic coefficient

$\begin{array}{ll}\mathrm{n}_{\mathrm{D}} & 1.72 \\ \mathrm{n}\left(\lambda_{\mathrm{p}}\right) & 1.69492 \\ \nu & 30 \\ \partial \mathrm{n} / \partial \mathrm{T} & -\end{array}$

$\partial s / \partial \mathrm{T}$

$\gamma$ calc

$\gamma$ meas

$\mathrm{n}_{2}$ calc

$\mathrm{n}_{2}$ meas

$\Delta \mathrm{B}$$$
-
$$$$
-
$$$$
-
$$$$
15.6 \times 10^{-20}
$$$$
6.29 \times 10^{-13}
$$

$\begin{array}{lll}\alpha & - & { }^{\circ} \mathrm{C}^{-1} \\ \mathrm{C}_{\mathrm{P}} & - & \mathrm{J} \cdot \mathrm{cm}^{-3} \cdot{ }^{\circ} \mathrm{C}^{-1} \\ \kappa & - & \mathrm{W} \cdot \mathrm{m}^{-1} \cdot{ }^{\circ} \mathrm{C}^{-1} \\ \mathrm{~T}_{\mathrm{y}} & - & { }^{\circ} \mathrm{C} \\ \mathrm{T}_{\mathrm{g}} & - & { }^{\circ} \mathrm{C}\end{array}$

\section{THERMAL PROPERTIES}

Thermal expansion coefficient

Specific heat at constant pressure

Thermal heat conductivity

Softening point

Transformation point

$\begin{array}{lll}\rho & 6.711 & \mathrm{~g} \cdot \mathrm{cm}^{-3} \\ \mathrm{~K}_{\mathrm{H}} & - & \mathrm{kg} \cdot \mathrm{mm}^{-2} \\ \mathrm{E} & - & \mathrm{kg} \cdot \mathrm{mm}^{-2} \\ \mathrm{G} & - & \mathrm{kg} \cdot \mathrm{mm}^{-2} \\ \sigma & - & \end{array}$

\section{SOLUBILITY}

Stability in water

Stability in acid

\section{-}

\section{REFERENCES and NOTES}

